CERN-TH/95-155

TAUP-2284-95

OSU RN309

hep-ph/9509312

\title{
Padé Approximants, Borel Transforms and Renormalons: the Bjorken Sum Rule as a Case Study
}

\author{
John Ellis \\ Theory Division, CERN, CH-1211, Geneva 23, Switzerland. \\ e-mail: johne@cernvm.cern.ch \\ and \\ Einan Gardi and Marek Karliner \\ School of Physics and Astronomy \\ Raymond and Beverly Sackler Faculty of Exact Sciences \\ Tel-Aviv University, 69978 Tel-Aviv, Israel \\ e-mail: gardi@post.tau.ac.il, marek@vm.tau.ac.il \\ and \\ Mark A. Samuel \\ Department of Physics, Oklahoma State University, \\ Stillwater, Oklahoma 74078, USA \\ e-mail: physmas@mvs.ucc.okstate.edu
}

\begin{abstract}
We prove that Padé approximants yield increasingly accurate predictions of higher-order coefficients in QCD perturbation series whose high-order behaviour is governed by a renormalon. We also prove that this convergence is accelerated if the perturbative series is Borel transformed. We apply Padé approximants and Borel transforms to the known perturbative coefficients for the Bjorken sum rule. The Padé approximants reduce considerably the renormalization-scale dependence of the perturbative correction to the Bjorken sum rule. We argue that the known perturbative series is already dominated by an infra-red renormalon, whose residue we extract and compare with QCD sum-rule estimates of higher-twist effects. We use the experimental data on the Bjorken sum rule to extract $\alpha_{s}\left(M_{Z}^{2}\right)=0.116_{-0.006}^{+0.004}$, including theoretical errors due to the finite order of available perturbative QCD calculations, renormalization-scale dependence and higher-twist effects.
\end{abstract}

CERN-TH/95-155

September 1995 


\section{Introduction}

Everybody interested in more precise quantitative tests of QCD, or in its place in some Grand Unified Theory, would welcome a more precise determination of the strong coupling strength $\alpha_{s}$ in some well-defined renormalization prescription, say $\overline{\mathrm{MS}}$, at some reference energy scale, say $M_{Z}$. Such determinations are normally made using perturbative QCD to interpret data, though lattice QCD may also become competitive once systematic effects are better controlled. Obtaining the desired level of precision using perturbative QCD requires calculations beyond the next-to-leading order. Several processes are calculated to high order in perturbative QCD [1], [2]. However, progress in the high-precision determination of $\alpha_{s}\left(M_{Z}\right)$ is hampered by the fact that the QCD perturbation series is expected to be asymptotic:

$$
S(x)=\sum_{n=0}^{\infty} c_{n} x^{n}, \quad x \equiv \frac{\alpha_{s}}{\pi}, c_{n} \simeq n ! K^{n} n^{\gamma}
$$

for some coefficients $K, \gamma$ [3]. Under these circumstances, how can one extract the most information from the QCD perturbation series, and obtain the best value of $\alpha_{s}=\pi x$ ? The usual answer is to calculate up to order $n_{o p t}: \Delta_{n} \equiv\left|c_{n} x^{n}\right|$ is minimized, and use the magnitude $\Delta_{n_{o p t}}$ of this minimum term as an estimator for the residual uncertainty.

In this paper we study whether it is possible to estimate $S_{\text {opt }}(x) \equiv \sum_{n=0}^{n_{o p t}} c_{n} x^{n}$ reliably without the labour of calculating all the perturbative coefficients $c_{n}: n \leq n_{\text {opt }}$, and comment on the possibility of summing over the higher-order terms $S_{a s y}(x) \equiv \sum_{n_{\text {opt }}}^{\infty} c_{n} x^{n}$ sufficiently reliably to reduce the magnitude of the residual uncertainty below $\Delta_{n_{o p t}}$. Our approach to these issues is based on Padé approximants, which are widely appreciated in other areas of physics, and which we have recently shown [4] can be used to predict higher-order perturbative QCD coefficients in agreement with exact calculations (where available) and with the effective charge method [5], [6].

In this paper, we present new results on the rate of convergence of Padé approximants for series of the form (四) expected in QCD. We also demonstrate that they reduce significantly the renormalization-scale dependence of the perturbative series for the Bjorken sum rule, and summarize a comparison with another technique for treating higher-order effects in perturbative QCD [7]. To go further, we transform to the Borel plane, where behaviours of the type (11) correspond to discrete renormalon singularities [3]. The Padé technique is a priori well adapted to locating such singularities, and we indeed prove that the convergence of the Padé approximants is accelerated for the Borel transform of a series such as (1). We apply this combined Padé-Borel technique to the calculated QCD perturbation series for the Bjorken sum 
rule, and show that it yields a leading infra-red renormalon pole close to the expected location in the Borel plane. Assuming this location, we extract its pole residue and use it to evaluate the possible magnitude of the infra-red renormalon ambiguity in the perturbative contribution to the Bjorken sum rule, which we argue is canceled by a corresponding ambiguity in the non-perturbative contribution. We use Padé summation, extracting $\alpha_{s}\left(M_{Z}^{2}\right)=0.116_{-0.006}^{+0.004}$ from the available polarized structure function measurements, including theoretical errors associated with renormalization-scale dependence and higher-twist effects. The accuracy of this result testifies to the utility of both Padé Approximants and the polarized structure function data.

\section{2 "Convergence" of Padé Approximants}

We denote Padé Approximants (PA's) to a generic perturbative QCD series $S(x)=\sum_{n=0}^{\infty} c_{n} x^{n}$ by

$$
[N / M]=\frac{a_{0}+a_{1} x+\ldots+a_{N} x^{N}}{1+b_{1} x+\ldots+b_{M} x^{M}}:[N / M]=S+O\left(x^{N+M+1}\right)
$$

i.e. the PA's are constructed so that their Taylor expansion up to and including order $N+M$ is identical to the original series. We have previously pointed out that the next term in the Taylor expansion of a $[N / M]$ PA typically provides increasingly accurate estimate $c_{N+M+1}^{e s t}$ of the next higher-order perturbative coefficient $c_{N+M+1}$ of the original series. In the following we refer to such estimates as Padé Approximant Predictions (PAP's).

Let us briefly restate the condition [8] for the convergence of PAP's. With $f(n) \equiv \ln c_{n}$ and $g(n) \equiv d^{2} f(n) / d n^{2} \quad$ (where the derivative with respect to $n$ is to be understood in a discrete sense), a sufficient condition for PAP convergence, $c_{N+M+1}^{e s t} \rightarrow c_{N+M+1}$, is $\lim _{n \rightarrow \infty} g(n)=0$. To quantify the rate of convergence, we introduce the quantity

$$
\epsilon_{n} \equiv \frac{c_{n} c_{n+2}}{c_{n+1}^{2}}-1=e^{g(n)}-1
$$

It is easy to check that $\epsilon_{n} \simeq 1 / n$ for an asymptotic series of the form (1). When the asymptotic behaviour of $\epsilon_{n}$ is known, it is possible to write down an asymptotic formula for the relative error $\delta_{[N / M]}$ in the PAP estimate $c_{N+M+1}^{e s t}$ of the perturbative coefficient $c_{N+M+1}$.

$$
\delta_{[N / M]} \equiv \frac{c_{N+M+1}^{\text {est. }}-c_{N+M+1}}{c_{N+M+1}}
$$

When $\epsilon_{n} \simeq 1 / n$, as is the case for an asymptotic series of the form (11), we have been able to demonstrate, for all values of $N$ and several values of $M$, that

$$
\delta_{[N / M]} \simeq-\frac{M !}{L^{M}}, \quad \text { where } L=N+M+a^{\prime} M+b .
$$


for some choice of numbers $a^{\prime}, b$. This implies that asymptotically

$$
\ln \left|\delta_{[M / M]}\right| \simeq-M\left[1+\ln \left(2+a^{\prime}\right)\right]
$$

We have verified that this formula is numerically accurate for simple series of the form (1) in which $c_{n}=n ! K^{n} n^{\gamma}$. Moreover, we have checked the prediction (6) for several different asymptotic series, including that for the QCD vacuum polarization $D$ function in the large- $N_{f}$ approximation [9], where it agrees numerically very well with the relative error reported in panel (a) of the figure in Ref. [4]. We note that the large- $N_{f} D$ function contains an infinite number of renormalon poles, which could in general provide important corrections to the leading-order formulae (10, 6). The fact that this is not the case supports the empirical utility of the PAP's even beyond the idealized analytical case (1).

In the previous paragraph, we have discussed the use of Padé approximants to estimate the next term in a given perturbative series, and, as we have seen, sufficient conditions for the convergence of such PAP's are known, so that the main open issue is their actual rate of convergence in practical applications. Next we discuss how to use PA's to estimate the "sum" of the perturbation series, which we term Padé Summation (PS).

It is important to note that convergence of the PAP's is largely independent of the "summability" of a given series. Thus, for example, the PAP method gives equally precise predictions for the next terms in the series $\sum_{0}^{\infty} n ! x^{n}$ and $\sum_{0}^{\infty} n !(-x)^{n}$, even though the latter is Borel summable, while the former is not.

The formal issues related to Padé Summation are less clear, especially because most perturbation series of practical interest are not Borel summable (since their Borel transforms have poles on the positive real axis). One well-defined prescription for defining the "sum" of such a series is the Cauchy principal value of the inverse Borel transform integral [10], so we ask whether PS "converges" to this prescription for the "sum" of the series. Such series are in general obtainable from functions with cuts on the positive real axis, with a toy example being provided by the simple asymptotic series $\sum_{0}^{\infty} n ! x^{n}$, which is a formal expansion of

$$
\int_{0}^{\infty} \frac{e^{-t}}{1-x t} d t=\frac{1}{x} \int_{0}^{\infty} \frac{e^{-y / x}}{1-y} d y
$$

We see from the representation on the right-hand side of (7) that this series corresponds in QCD language to a single simple infrared renormalon pole, whilst the left-hand side of (7) exhibits a cut on the positive real axis. Figure 1 exhibits the errors with respect to the Cauchy principal value of the integral (7) of conventional partial sums and PA's to the series $\Sigma_{0}^{\infty} n ! x^{n}$ 
for the case $x=0.1$ for which $n_{\text {opt }}=9$. We see that the first few PA's have relative errors that are considerably smaller than those of the partial sums. The best PA has an error comparable to $\Delta n_{\text {opt }}$, despite using as input a number of terms that is less than $n_{\text {opt }}$. However, we see that higher-order PA's exhibit relative errors that are irregular, and may even be less accurate than the conventional partial sums. This is because the Padé method mimics the cut on the lefthand side of (7) with an ever-denser set of poles, the $[N / M]$ PA's having typical separations $\Delta x=\mathcal{O}(1 / M)$. This makes it ever more difficult to avoid nearby poles when the PA's are evaluated at any fixed value of $x$, and therefore to define "convergence" of the PS procedure. However, PA's of sufficiently high order are again much more accurate than the partial sums, which are blowing up because of the renormalon singularity.

As also seen in Figure 1, it is possible to improve on the simple PA's. One way of smoothing out the irregularities associated with nearby poles is to evaluate the real parts of the $[N / M]$ PA's off the real axis] at $x+i \epsilon$. It is a theorem [11] that this smoothed PS prescription converges to the Cauchy principal value in the cut plane $|\arg z|>0$, if the weight in the integral over the positive real axis is positive. Another approach is simply to remove from the PA any spurious contribution of a nearby pole. Yet another is to make a Taylor expansion of the PA and truncate it at order $n_{\text {opt }}$. We have developed [12] criteria for deciding which of these is better for any given case, and the application to our toy example is also shown in Figure 1, designated by "combined method". Since the investigation of these techniques is still in progress, we do not discuss them further in this paper, deferring this to a future publication [12], where a detailed description will be given, together with physical applications.

\section{Application to the Bjorken Sum Rule}

We now discuss a concrete application of PA's to a perturbative QCD series, namely that for the Bjorken sum rule, which takes the following form in the $\overline{\mathrm{MS}}$ renormalization prescription [13], [1]:

$$
\int_{0}^{1}\left[g_{1}^{p}\left(x, Q^{2}\right)-g_{1}^{n}\left(x, Q^{2}\right)\right]=\frac{1}{6}\left|g_{A}\right| f(x): \quad f(x)=1-x-3.58 x^{2}-20.22 x^{3}+\ldots+(H T)
$$

for $N_{f}=3$, as relevant to the $Q^{2}$ range of current experiments, where $x=\alpha_{s}\left(Q^{2}\right) / \pi$, the dots represent uncalculated higher orders of perturbation theory, and $(H T)$ denotes higher-twist terms. The perturbative series in (8) is expected to be dominated by renormalons in large

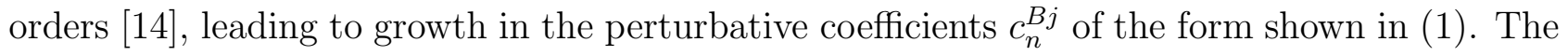

\footnotetext{
${ }^{1}$ We thank A.A. Migdal for pointing this out.
} 
PA's to the series (8) yield the following predictions for the next term

$$
\begin{aligned}
& c_{4[P A]}^{B j} \approx-111 \quad([1 / 2] \quad \mathrm{PA}) \\
& c_{4[P A]}^{B j} \approx-114 \quad([2 / 1] \mathrm{PA})
\end{aligned}
$$

in this series.

In order for these PAP's to be useful, it is important to estimate the errors involved. The asymptotic error estimate given in eq. (5) requires as input values of $a^{\prime}$ and $b$, which are not known a priori. Experience with many series shows that typically $-1 \lesssim a^{\prime} \lesssim 0$ and $b \approx 0$. With $a^{\prime}=0$ and $b=0$ we obtain a ballpark estimate

$$
\delta_{[1 / 2]} \simeq-2 / 9 ; \quad \delta_{[2 / 1]} \simeq-1 / 3
$$

In a previous paper [4], we used a different method to estimate the errors of the Padé prediction (9), obtaining $c_{4[P A]}^{B j}=-112 \pm 33$ as the error-weighted average of the $[1 / 2]$ and $[2 / 1]$ approximants. We also pointed out that this prediction is close to an estimate made using the Effective Charge Method (ECH):

$$
c_{4[E C H]}^{B j} \simeq-130
$$

We now note further that even though the error estimates (10) are in principle expected to hold only asymptotically, in practice (9) and (11) are consistent with each other within these estimates. We take this as an indication that the true value of $c_{4}^{B j}$ is likely to be in the range predicted by the PAP and ECH methods. Moreover, as we shall show in section 4, it seems that the perturbation series in (8) is already dominated by a single infrared renormalon, in which case the quantities (10) are no longer "statistical" errors, but fractional corrections to be subtracted from (9), improving the concordance with the ECH estimate (11).

The next step is to apply the PS procedure to estimate the complete correction function $f(x)$ in (8). Figure 2 compares $f^{[2 / 2]}(x)$, the [2/2] PS estimate of $f(x)$ (obtained using the ECH value (11) of the fourth-order perturbative coefficient),

$$
f^{[2 / 2]}(x)=\frac{1-8.805 x+11.974 x^{2}}{1-7.805 x+7.753 x^{2}}
$$

with the [1/2], [2/1] PS's and with the partial sums of the perturbative series up to order $x^{3}$ and $x^{4}$ (the latter also taken from (11) ). We see that the different PS's are numerically quite stable in the range $x \lesssim 0.1$ of relevance to present experiments, which is related to the fact that the nearest poles are some distance away $(x=0.18$ for the $[2 / 1] \mathrm{PA}, x=-3.41$ and $x=0.18$ 
for the $[1 / 2] \mathrm{PA}$, and $x=0.15$ and $x=0.86$ for the [2/2] PA). This means that the "combined method" for smoothing of PA's described at the end of the previous section is not necessary.

We now check the reliability of the PS estimates of $f(x)$ in two different ways, first by checking their renormalization-scale dependences. Figure 3 compares the values of $\alpha_{s}\left(Q^{2}\right)$ estimated from a fixed value of $f(x)$ at $Q^{2}=3 \mathrm{GeV}^{2}$, when one renormalizes at scales $\mu$ in the range between $Q / 2$ and $2 Q$, using the PS or the third- or fourth-order partial sum. We see that the value of $\alpha_{s}\left(Q^{2}\right)$ extracted from the PS is indeed much less $\mu$-dependent than the values extracted using the partial sums [15], consistent with our belief that the PS's provides a reliable estimate of the full correction factor $f(x)$, which should be independent of $\mu$. In particular, the $\mu$-dependence of the [2/2] PS is small compared with other possible sources of theoretical error.

We have also compared the PS's with results based on the BLM treatment of the perturbative series in the $\overline{\mathrm{MS}}$ scheme, in which the growing higher-order coefficients are absorbed into the scales $Q^{2}$ at which $x=\alpha_{s}\left(Q^{2}\right) / \pi$ is evaluated in each of the lower-order terms. As described elsewhere [12], we find that our PS procedure agrees very well with the BLM procedure when applied to the perturbative series for the Bjorken sum rule, adding further support to our contention that the PA's may indeed accelerate usefully the convergence of perturbative QCD series, as suggested by the general arguments of Section 2.

\section{Padé Approximants in the Borel Plane}

If one knows the asymptotic behaviour of the series under study, one can go further. In particular, if the perturbative coefficients diverge as in (1), which is believed to be the case in perturbative QCD, corresponding to a discrete set of renormalons [3], it is useful to consider the Borel transform of the series $S(x)$ in eq. (囵):

$$
\tilde{S}(y) \equiv \sum_{n=0}^{\infty} \tilde{c}_{n} y^{n}: \tilde{c}_{n}=\frac{c_{n+1}}{n !}\left(\frac{4}{\beta_{0}}\right)^{n+1} ; \quad \beta_{0}=\left(33-2 N_{f}\right) / 3
$$

If the Borel transform $\tilde{S}(y)$ indeed has a discrete set of renormalon singularities $r_{k} /\left(y-y_{k}\right)^{P}$, where the $r_{k}$ 's are the residues, PA's in the Borel plane are a priori well suited to find them. Indeed, if there is a finite set of renormalon singularities, as occurs for the Bjorken sum rule series in the large- $N_{f}$ approximation, higher-order PA's will be exact. In general, the removal of the $n$ ! factors in the coefficients (13) means that the corresponding quantity measuring the 
rate of convergence of the Padé prediction for the next term is

$$
\tilde{\epsilon}_{n}=\frac{\tilde{c}_{n} \tilde{c}_{n+2}}{\tilde{c}_{n+1}^{2}} \simeq \frac{1}{n^{2}},
$$

which is much smaller than the previous $\epsilon_{n} \simeq 1 / n(3)$. This means that the relative error $\tilde{\delta}_{[N, M]}$ in the PA of the Borel-transformed series is also much smaller than (5) for the original series,

$$
\tilde{\delta}_{[M / M]} \simeq-\frac{(M !)^{2}}{L^{2 M}}
$$

corresponding asymptotically to

$$
\ln \left|\tilde{\delta}_{[M / M]}\right| \simeq-2 M\left[1+\ln \left(2+a^{\prime}\right)\right]
$$

We have also checked this prediction for several different asymptotic series, including that for the QCD vacuum polarization $D$ function in the large- $N_{f}$ approximation [9], which has a discrete infinity of renormalon poles. The prediction (16) again agrees numerically very well with the relative error reported in panel (b) of the figure in Ref. [4], which is much smaller than that for the naive PA in panel (a). Again, the success of the prediction (15) gains significance from the fact that the large- $N_{f}$ calculation exhibits an infinity of renormalon poles, indicating that the Borel PA's are useful in the real world, and not only in idealized simplified situations.

We now apply this combined Borel/Padé technique to the QCD perturbation series (8) for the Bjorken sum rule. With the normalization of the Borel variable $y$ implicitly defined through eq. (13), the [2/1] PA to the Borel transform of (8) has a pole at $y=1.05$ with residue $r=$ 0.98, as seen in Fig. 4. The appearance of a pole near $y=1$ is encouragingly consistent with the exact large- $N_{f}$ calculations [14, which yield poles at $y= \pm 1, \pm 2$. In the $\overline{\mathrm{MS}}$ prescription that we are using, the residues of these poles contain factors exp[5y/3]. Therefore, it is not surprising that an infra-red renormalon pole at $y=1$ emerges more clearly than an ultraviolet renormalon pole at $y=-1$. Prima facie, the message of this analysis is that the calculated Bjorken series is already dominated by the expected leading infra-red renormalon.

Encouraged by this success, we have made fits to the Borel transform of the Bjorken series with varying numbers of poles whose locations are fixed in accordance with theoretical expectations. The residues found in these various fits are also plotted in Fig. 4, where we see the following points:

(i) the residue of the $y=1$ pole is consistently found to be positive and around unity,

(ii) the residue of the $y=-1$ pole is much smaller, and consistent with zero, 
(iii) there is room for a second pole at $y=2$, but it is not possible to disentangle this from a higher-lying pole.

We now discuss the possible implications of this Borel/Padé exercise for phenomenology. First, we note that the dominance by a single infrared renormalon pole indicates that, as already remarked, the fractional errors (10) should be subtracted from the naive estimates (9), bringing them into better agreement with the ECH estimate (11):

$$
c_{4[P A]}^{B j} \simeq-136,-152
$$

from the $[1 / 2]$ and $[2 / 1]$ PA's respectively. Secondly, it is well known that the magnitude of the residue $r_{1}$, of the $y=1$ pole corresponds to a possible renormalon ambiguity $\pm \pi r_{1}$ relative to the Cauchy principal value discussed previously. This is also shown for our toy series in Fig. 1. Taking $r_{1}$ for the Bjorken series from Fig. 4, we find an ambiguity

$$
\Delta\left(\Gamma_{1}^{p}-\Gamma_{1}^{n}\right)= \pm \frac{\left|g_{A}\right|}{6} 0.98 \pi \frac{\Lambda^{2}}{Q^{2}}
$$

in the perturbative contribution to the Bjorken sum rule. Numerically, with $\Lambda=250 \pm 50 \mathrm{MeV}$, this corresponds to $\pm 0.040 \pm 0.016 \mathrm{GeV}^{2} / Q^{2}$, which is to be compared with previous QCD sum rule higher-twist estimates that yield [16]

$$
\Delta_{H T}\left(\Gamma_{1}^{p}-\Gamma_{1}^{n}\right)=-\frac{0.02 \pm 0.01}{Q^{2}}
$$

(see also the discussion pertaining to eq. (7) in Ref. [17], and a recent estimate in Ref. [18] ). We note that the order of magnitude of the renormalon ambiguity we find (18) is close to the higher-twist calculation (19).

Since the full QCD prediction for any physical quantity must be unique, the renormalon ambiguity must be cancelled by a corresponding ambiguity in the definition of the higher-twist term. For this reason we do not interpret the renormalon ambiguity as leading directly to an ambiguity in $\alpha_{s}$, but rather use the uncertainty in the higher twist term (19).

\section{$5 \quad$ Extraction of $\alpha_{s}$ from Bjorken Sum Rule Data}

We conclude this paper by extracting [17] $\alpha_{s}\left(M_{Z}^{2}\right)$ from data on the Bjorken sum rule, including

a discussion of theoretical errors. We combine the available experimental evaluations of $\Gamma_{1}^{p, n}\left(Q^{2}\right)$ to obtain

$$
\Gamma_{1}^{p}\left(3 \mathrm{GeV}^{2}\right)-\Gamma_{1}^{n}\left(3 \mathrm{GeV}^{2}\right)=0.164 \pm 0.011
$$


where we have evolved all the experimental results [19]-21] to the common reference scale $Q^{2}=3 \mathrm{GeV}^{2}$. Evolution of the proton data from 10 to $3 \mathrm{GeV}^{2}$ is based on the analysis in Ref. [20]. The corresponding evolution of the deuteron data is based on the proton data, combined with the expected $Q^{2}$ dependence of the Bjorken sum rule, with $\alpha_{s}\left(Q^{2}\right)$ needed as input determined via iteration, in a self-consistent way. Evolution to $3 \mathrm{GeV}^{2}$ from values of $Q^{2}$ different from $10 \mathrm{GeV}^{2}$ was treated in a linear approximation to the full dependence of the data on $1 / \log \left(Q^{2}\right)$, which is adequate for this purpose.

The range of $f(x)$ corresponding to (20) is shown as a vertical error bar in Figure 2. We use the $[2 / 2]$ PS (12) to obtain: $\alpha_{s}\left(3 \mathrm{GeV}^{2}\right)=0.328_{-0.037}^{+0.026}$. We evolve this up to $M_{Z}^{2}$ by numerical integration of the three-loop $\beta$ function [2], locating the $b$-quark threshold in the $\overline{\mathrm{MS}}$ scheme at $m_{b}=4.3 \pm 0.2 \mathrm{GeV}$ [22], and using the three-loop matching conditions of ref. [23], to find

$$
\alpha_{s}\left(M_{Z}^{2}\right)=0.119_{-0.005}^{+0.003} \pm \ldots \quad
$$

where the $\pm \ldots$ in (21) recalls that theoretical errors remain to be assigned.

There is a theoretical error associated with the spread in the different evaluation procedures shown in Fig. 2, which we estimate from the difference between the $[2 / 2]$ and $[1 / 2],[2 / 1]$ PS's to be $\Delta_{\text {proc }} \alpha_{s}\left(3 \mathrm{GeV}^{2}\right)= \pm 0.014$, corresponding to

$$
\Delta_{\text {proc }} \alpha_{s}\left(M_{Z}^{2}\right)= \pm 0.002
$$

Another way of looking at the theoretical error uses the $\mu$-dependence of the $[2 / 2]$ PS shown in Fig. 3 to estimate $\Delta_{\mu} \alpha_{s}\left(3 \mathrm{GeV}^{2}\right)= \pm 0.009$, corresponding to

$$
\Delta_{\mu} \alpha_{s}\left(M_{Z}^{2}\right)= \pm 0.001
$$

from varying $\mu$ between $Q / 2$ and $2 Q$. Both (22) and (23) are estimates of the uncertainty in $\alpha_{s}\left(M_{Z}^{2}\right)$ due to our uncertainty in the functional form of the QCD correction factor $f(x)$, so that it could be regarded as double counting to include them both. Nevertheless, to be conservative we will add them in quadrature.?

We also include a shift and error in the determination of $\alpha_{s}\left(Q^{2}\right)$ inferred from the estimated range (19) of the higher-twist correction: $\Delta_{H T} \alpha_{s}\left(3 \mathrm{GeV}^{2}\right)=-0.024 \pm 0.014$, corresponding to

$$
\Delta_{H T} \alpha_{s}\left(M_{Z}^{2}\right)=-0.003 \pm 0.002
$$

\footnotetext{
${ }^{2}$ We have also considered possible systematic theoretical errors due to uncertainties in the evolution of $\alpha_{s}$ up to $M_{Z}$, including unknown higher-order terms in the QCD $\beta$-function, the uncertainty in $m_{b}$, and freedom in treating the heavy-flavour threshold [23]. They contribute an error in $\alpha_{s}\left(M_{Z}, 2\right)$ which is much less than \pm 0.001 .
} 
Combining (21), (22), (23), (24), we extract

$$
\alpha_{s}\left(M_{Z}^{2}\right)=0.116_{-0.005}^{+0.003} \pm 0.003
$$

where the first errors are the experimental errors in (21), and the second errors are the sums in quadrature of the errors in (22), (23), (24).

Our final value of $\alpha_{s}\left(M_{Z}^{2}\right)$ (25) is compatible with the central value extracted from compilations of previous measurements [24], and has an error which is competitive. As well as the experimental error, we have included motivated estimates of a number of theoretical errors, using information obtained from our study of Padé approximants. We believe that this exercise demonstrates the utility of Padé Approximants in QCD, and the value of polarized structure function data for determining $\alpha_{s}\left(M_{Z}^{2}\right)$.

\section{Acknowledgments}

We thank David Atwood, Bill Bardeen, Stan Brodsky, Georges Grunberg, Sasha Migdal and Al Mueller for useful discussions. This research was supported in part by the Israel Science Foundation administered by the Israel Academy of Sciences and Humanities, and by a Grant from the G.I.F., the German-Israeli Foundation for Scientific Research and Development. It was also in part supported by the US Department of Energy under Grant No. DE-FG02-94ER40852.

\section{References}

[1] S.A. Larin, F.V. Tkachev and J.A.M. Vermaseren, Phys. Rev. Lett. 66(1991)862; S.A. Larin and J.A.M. Vermaseren, Phys. Lett. B259(1991)345.

[2] S.G. Gorishny, A.L. Kataev and S.A. Larin, Phys.Lett. B259(1991)144; L.R. Surguladze and M.A. Samuel, Phys.Rev.Lett. 66(1991)560; S.G. Gorishny, A.L. Kataev and S.A. Larin, Standard Model and Beyond: from LEP to UNK and LHC, ed. by S. Dubnicka et al. (World Scientific, Singapore, 1991) p.299; O.V. Tarasov, A.A. Vladimirov and A.Yu. Zharkov, Phys.Lett. 93B(1980)429.

[3] A. Mueller Nucl.Phys. B250(1985)327; A.I. Vainshtein and V.I. Zakharov, Phys.Rev.Lett. $73(1994) 1207$.

[4] M.A. Samuel, J. Ellis and M. Karliner, Phys. Rev. Lett. 74(1995)4380. 
[5] G. Grunberg, Phys.Lett. 95B(1980)70, E - ibid. 110B(1982)501; Phys.Rev. D29(1984)2315; P.M. Stevenson, Phys.Rev. D23(1981)2916;

[6] A. L. Kataev and V. V. Starshenko, Mod.Phys.Lett. A10(1995)235.

[7] S.J. Brodsky, G.P. Lepage and P.M. Mackenzie, Phys.Rev. D51(1995)3652; see also S.J. Brodsky and H. J. Lu, Precision Tests of Quantum Chromodynamics and the Standard model, SLAC-PUB-95-6937, hep-ph/9506322, and references therein.

[8] M.A. Samuel, G. Li and E. Steinfelds, Phys. Rev. E51(1995)3911; M.A. Samuel and S.D. Druger, Intl. J. Th. Phys. 34(1995)903.

[9] C.N. Lovett-Turner and C.J.Maxwell Nucl. Phys. B432(1994)147.

[10] G. Grunberg, Phys. Lett. B325 (1994) 441; see also V.A. Fateev, V.A. Kazakov and P.B. Wiegmann, Nucl.Phys. B424(1994)505 for a 2D example where this prescription is exact.

[11] C.M. Bender and S.A. Orszag, Advanced Mathematical Methods for Scientists and Engineers, McGraw-Hill, 1978, sec. 8.6 and Prob. 8.59 .

[12] J. Ellis, E. Gardi, M. Karliner and M. Samuel, to be published.

[13] J. Bjorken, Phys.Rev. 148(1966)1467; Phys.Rev. D1(1970)1376.

[14] C.N. Lovett-Turner and C.J. Maxwell, All Orders Renormalon Resummations for Some QCD Observables, Durham preprint DTP-95-36, hep-ph/9505224.

[15] P.A. Rạczka, Z. Phys C65(1995)481, hep-ph/9506462.

[16] I.I. Balitsky, V.M. Braun and A.V. Kolesnichenko, Phys.Lett. B242(1990)245; erratum: ibid, B318(1993)648. B. Ehrnsperger, A. Schaefer and L. Mankiewicz, Phys.Lett. B323(1994)439; G.G. Ross and R.G. Roberts, Phys.Lett. B322(1994)425; E. Stein et al., Phys.Lett. B343(1995)369; E. Stein et al., Phys.Lett. B353(1995)107.

[17] J. Ellis and M. Karliner, Phys.Lett. 341(1995)397.

[18] V.M. Braun, QCD Renormalons and Higher Twist Effects, Proc. Moriond 1995, hep$\mathrm{ph} / 9505317$. 
[19] SLAC-Yale E80 Coll., M.J. Alguard et al., Phys.Rev.Lett. 37(1976)1261; 41(1978)70; SLAC-Yale Coll., G. Baum et al., Phys.Rev.Lett. 45(1980)2000; SLAC-Yale E130 Coll., G. Baum et al., Phys.Rev.Lett. 51(1983)1135; EMC Coll., J. Ashman et al., Phys.Lett. B206(1988)364, Nucl.Phys. B328(1989)1; SMC Coll., B. Adeva et al., Phys.Lett. B302(1993)533; E142 Coll., P.L. Anthony et al., Phys.Rev.Lett. 71(1993)959; SMC Coll., D. Adams et al., Phys.Lett. B329(1994)399; E143 Coll., K. Abe et al., Phys.Rev.Lett. 74(1995)346, Phys.Rev.Lett. 75(1995)25; SMC Coll., D. Adams et al., A New Measurement of the Spin Dependent Structure Function $g_{1}(x)$ of the Deuteron, CERN-PPE-95-097 (June 1995).

[20] P. Grenier, Ph.D. dissertation (1995) and private communication.

[21] Y. Roblin, Ph.D. dissertation (1995).

[22] Particle Data Group, Review of Particle Properties, Phys.Rev. D50(94)1173.

[23] W. Bernreuther, Z. Phys. C20(1983)331.

[24] S. Bethke, Aachen preprint PITHA-95-14 (1995), to appear in Proc. 30-th Rencontre de Moriond, QCD and High-Energy Hadronic Interactions. 


\section{FIGURE CAPTIONS}

Fig. 1. The relative errors between partial sums of the series $S(x)=\Sigma n ! x^{n}$ and the Cauchy principal value of the series (solid line) is compared with the relative errors of Padé Sums (dotted line). We see that the relative errors of the Padé Sums are smaller than those of the partial sums in low orders, fluctuate in an intermediate régime, and are again more accurate than the partial sums in higher orders. The fluctuations are associated with nearby poles in the Padé Sums, that may be treated by the "combined method" mentioned in the text, shown as the dashed line.

Fig. 2. Different approximations to the Bjorken sum rule correction factor $f(x)$, thirdorder and fourth-order perturbation theory, [1/2], [2/1] and [2/2] Padé Sums (12), are compared. Also shown as a vertical error bar is the value of $f(x)$ we extract from the available polarized structure data (20).

Fig. 3. The scale dependence of $\alpha_{s}\left(3 \mathrm{GeV}^{2}\right)$ obtained from a fixed value $f(x)=\left(6 / g_{A}\right) \times$ $0.164=0.783$, (cf. eq. (20) ), for $Q / 2<\mu<2 Q$, using the naive third- and fourth-order perturbative series and the [1/2], [2/1] and [2/2] PS's.

Fig. 4. The locations and residues of poles in the $[2 / 1] \mathrm{PA}$ and in rational-function fits to the Borel transform of the first four terms in the perturbation series for the Bjorken sum rule. We note that the location of the lowest-lying infrared renormalon pole is estimated accurately by Padé Approximants in the Borel plane, and that its residue is stable in the different fits. 


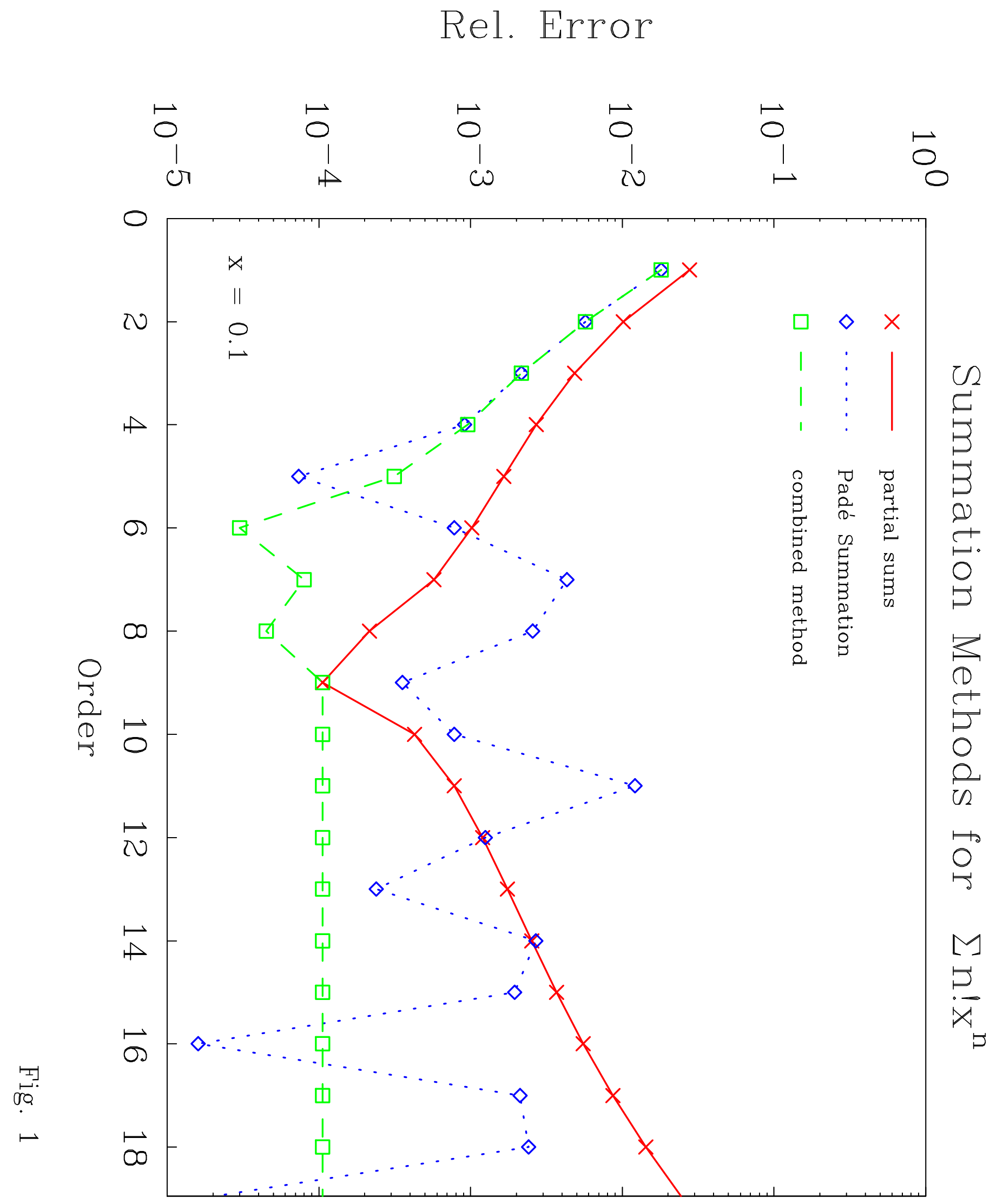


这

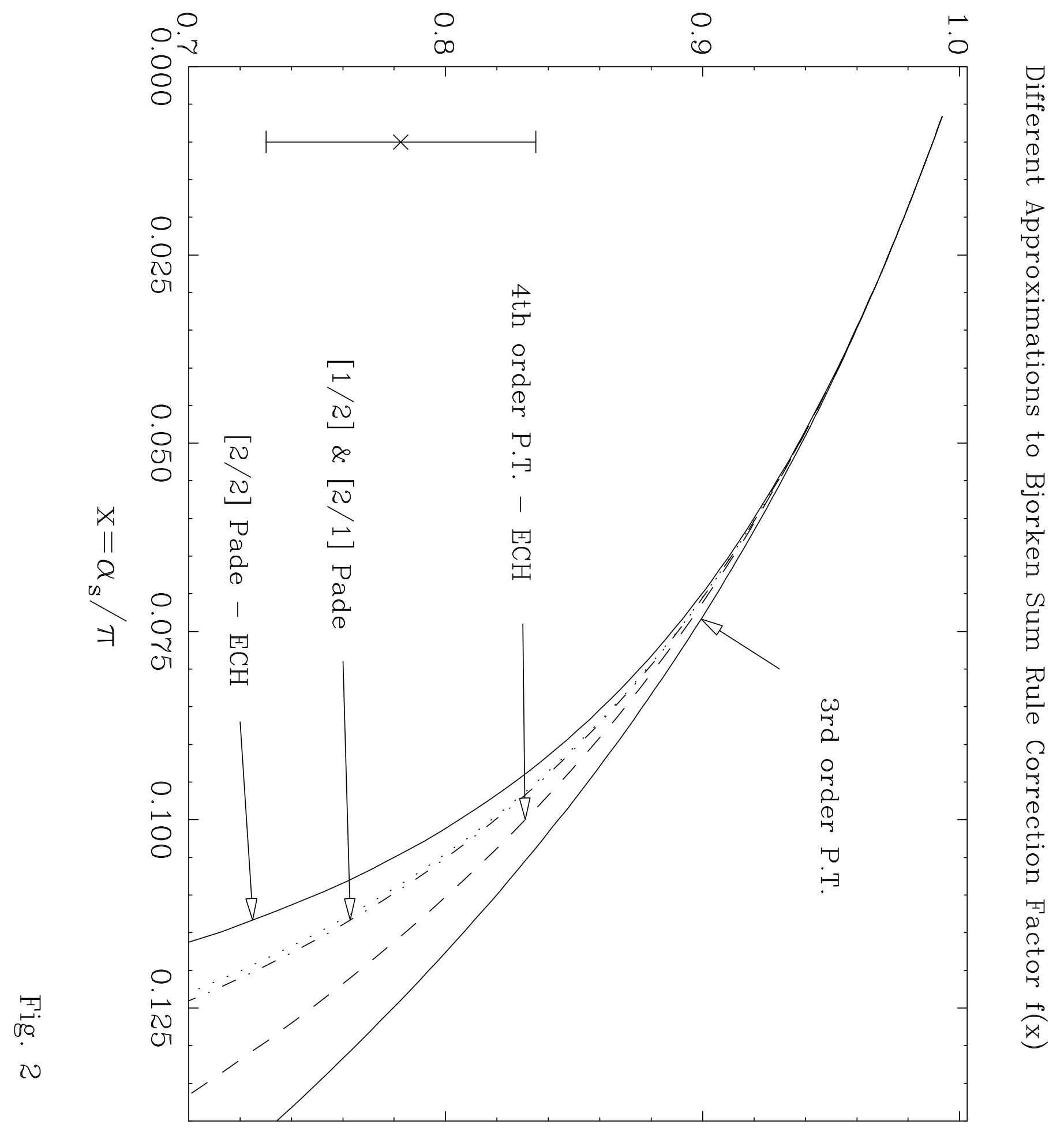




$$
\alpha_{\mathrm{s}}^{\overline{\mathrm{MS}}}\left(\mu, \mathrm{Q}^{2}=3.0 \mathrm{GeV}^{2}\right)
$$

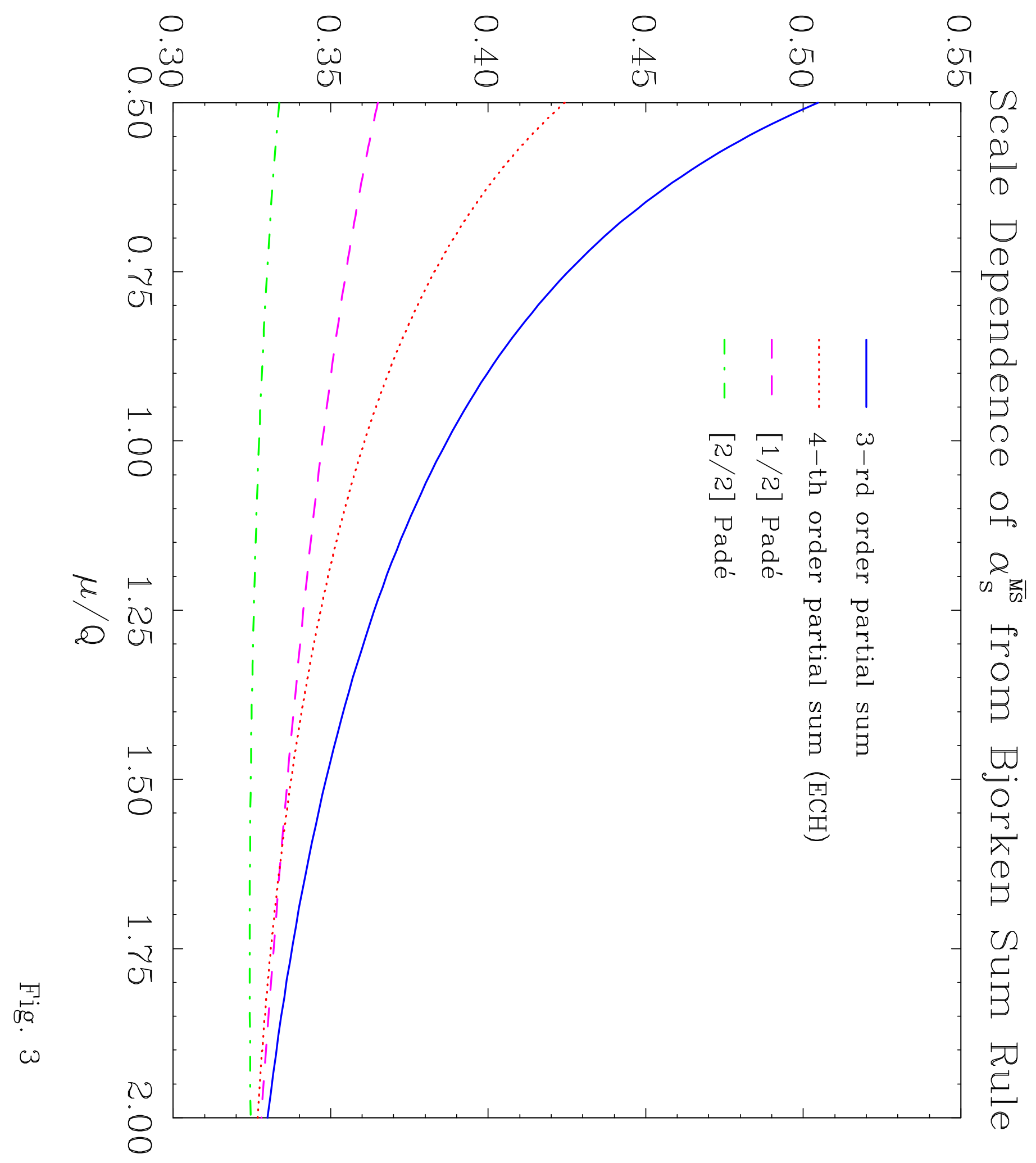


Residue

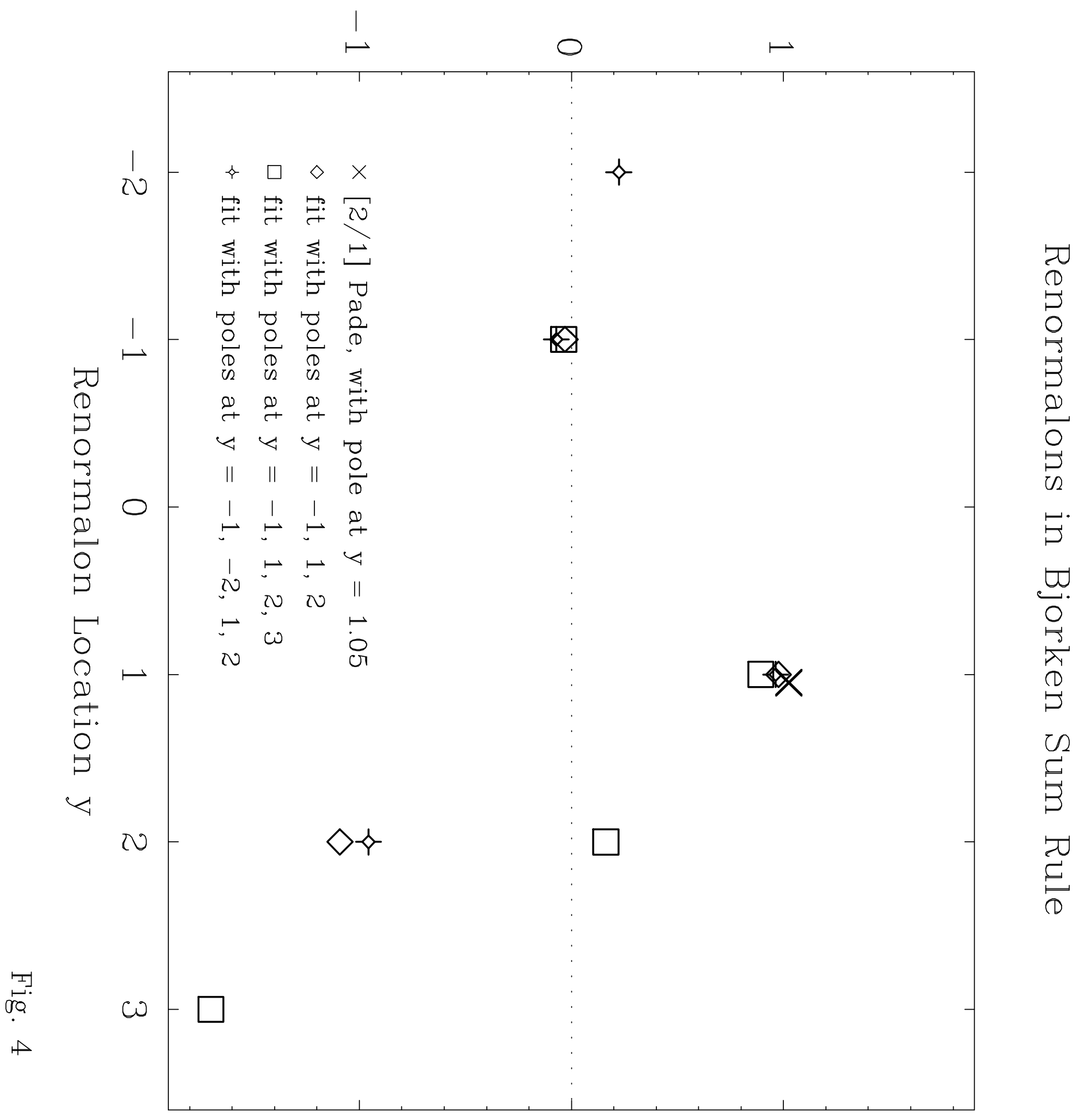

